\title{
Endobronchial Valves in the Management of Recurrent Haemoptysis
}

\author{
Coenraad F.N. Koegelenberg Johannes W. Bruwer Chris T. Bolliger
}

Division of Pulmonology, Department of Medicine, Stellenbosch University and Tygerberg Academic Hospital, Cape Town, South Africa

\section{Established Facts}

- Haemoptysis remains a life-threatening complication of bronchiectasis and other structural lung diseases.

- Endoscopic treatment modalities for life-threatening haemoptysis in patients unresponsive to medical interventions and/or in patients deemed inoperable are limited.

\section{Novel Insights}

- Endobronchial valves may represent feasible blockade devices for the management of recurrent haemoptysis.

- Endobronchial valves may improve pulmonary function in patients with bronchiectasis secondary to tuberculosis.

\section{Key Words}

Bronchiectasis · Endobronchial valves · Haemoptysis

\begin{abstract}
Minimally invasive treatment modalities for life-threatening haemoptysis in patients unresponsive to medical interventions and/or in patients deemed functionally inoperable are limited. We describe the implantation of endobronchial valves in a patient with recurrent haemoptysis, which presents both a novel indication for the use of these devices and a novel intervention for haemoptysis. Our patient is a 30-year-old male who developed bilateral upper lobe aspergillomata following previous pulmonary tuberculosis. The patient had a history of multiple hospitalisations for life-
\end{abstract}

\section{KARGER}

E-Mail karger@karger.com www.karger.com/res threating haemoptysis despite repeated bronchial artery embolisations. He was deemed to be inoperable given the bilateral nature of his disease and very poor pulmonary reserves. We proceeded to identify the segments involved with the aid of computed tomography reconstruction and implanted 3 endobronchial valves. Our patient remained haemoptysis free for 6 months and experienced no stentrelated complications. Moreover, he was subsequently employed as a manual labourer and showed significant improvements in his functional capacity. Endobronchial valves may therefore represent a viable medium-term treatment option as a blockade device in patients unresponsive to

\section{Dr. Chris T. Bolliger is deceased.}


medical interventions and/or in patients deemed functionally inoperable. Prospective studies are indicated to better delineate the role of endobronchial valves in this setting.

(c) 2013 S. Karger AG, Basel

\section{Introduction}

Haemoptysis remains a feared and potentially fatal complication in patients with structural lung disease [1]. Bronchial arterial embolisation is an effective intervention offered to patients who do not respond to acute medical management [1]. It provides acute control in the overwhelming majority of cases, but rebleeding is seen in approximately half of the treatment-responsive patients, particularly in the presence of bronchiectasis secondary to tuberculosis (TB) and aspergillomata [1-3]. Surgical resection remains the only curative option in the majority of cases, provided it is technically feasible and the patient has the functional reserve to withstand pulmonary resection [4].

Various endoscopic procedures have been utilised to control haemoptysis both in the acute setting and as a bridge to surgery $[5,6]$. These include the placement of balloon catheters, laser photocoagulation and electrocautery $[5,6]$. All these procedures are, however, of limited medium-term benefit, particularly if surgical resection is not an option $[5,6]$. The use of topical haemostatic tamponade therapy or blockade devices, which could be deployed and left in place permanently or be removed (e.g. a silicone spigot), are relatively novel therapeutic approaches that have been shown to be beneficial, at least in the short term [5-7].

Endobronchial valves were primarily developed for patients with severe emphysema and significant hyperinflation to facilitate deflation of the overinflated emphysematous parts of the lung [8-10]. Other indications for these valves include the treatment of bronchopleural fistulas and post-operative air leaks $[11,12]$.

We describe herein the successful use of endobronchial valves in a patient with poor functional reserve and recurrent life-threatening haemoptysis unresponsive to medical interventions.

\section{Case Report}

A 30-year-old male presented with recurrent episodes of lifethreatening haemoptysis. He reported poor exercise tolerance and a chronic cough, but denied any constitutional symptoms sugges-

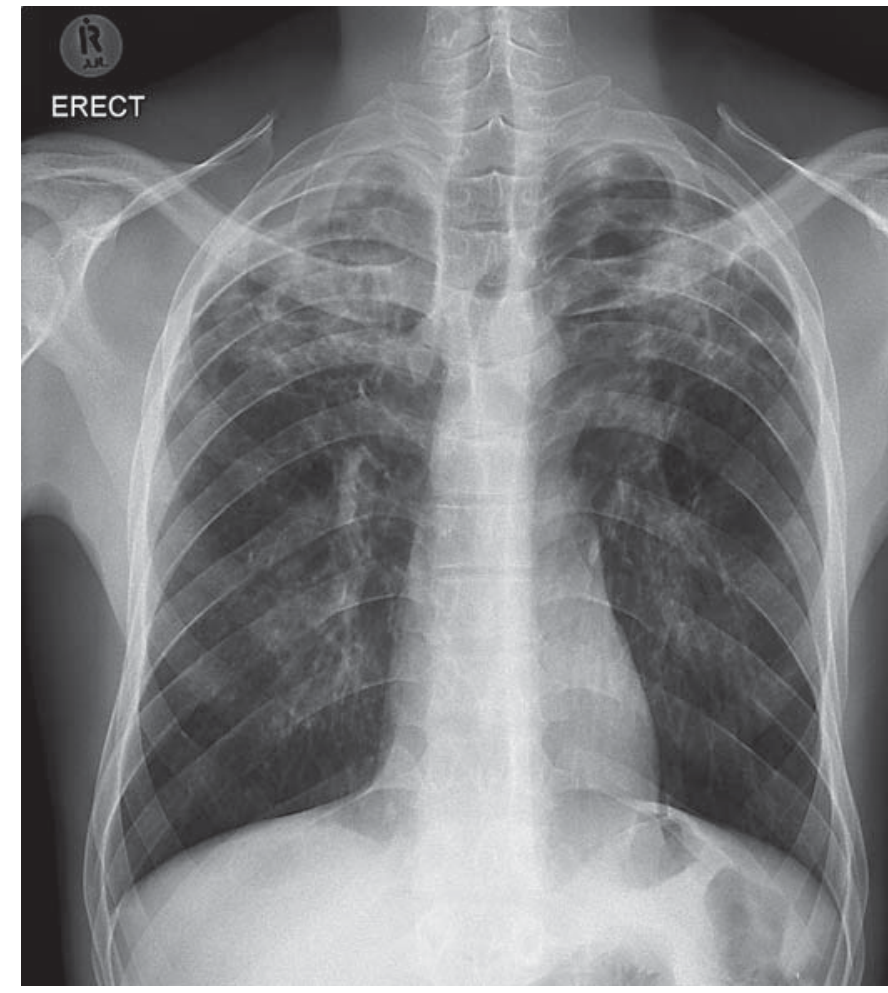

Fig. 1. The patient's chest radiograph showed marked bilateral UL post-TB bronchiectasis with multiple cavities.

tive of active pulmonary TB. The patient was known to be HIV positive with a preserved CD4 count and was previously fully treated for pulmonary TB. Previously, he had been hospitalised on 2 occasions for life-threatening haemoptysis, and red cell transfusion and bronchial artery embolisation were required during both admissions. He experienced ongoing low-grade haemoptysis at the time of his assessment at our clinic.

The patient's chest radiograph (fig. 1) and CT of the chest (fig. 2) revealed marked post-TB structural lung disease with prominent upper lobe (UL) bronchiectasis and numerous cavities with intracavitary bodies suggestive of aspergillomata. These findings were static and also present on radiographs taken 18 months prior to the present evaluation. He was deemed not to be a candidate for surgery given the bilateral nature of his disease and very poor pulmonary reserve $\left[\mathrm{FEV}_{1}=0.78\right.$ liters $(19.3 \%), \mathrm{FVC}=0.95$ liters $(21.6 \%)$ and 6 - $\mathrm{min}$ walking distance $=78 \mathrm{~m}]$.

After obtaining consent from the patient as well as our local ethics committee, we proceeded to identify the segments involved using CT reconstruction software. We subsequently placed 3 endobronchial valves (IBV Valve System ${ }^{\circledR}$, Olympus Respiratory America, Redmond, Wash., USA) with the aid of a flexible bronchoscope in the right UL anterior segment as well as the apical and anterior segments of the left UL, respectively. The chest X-ray (fig. 3) taken after the procedure confirmed adequate stent placement and deployment with partial volume loss of both ULs. 

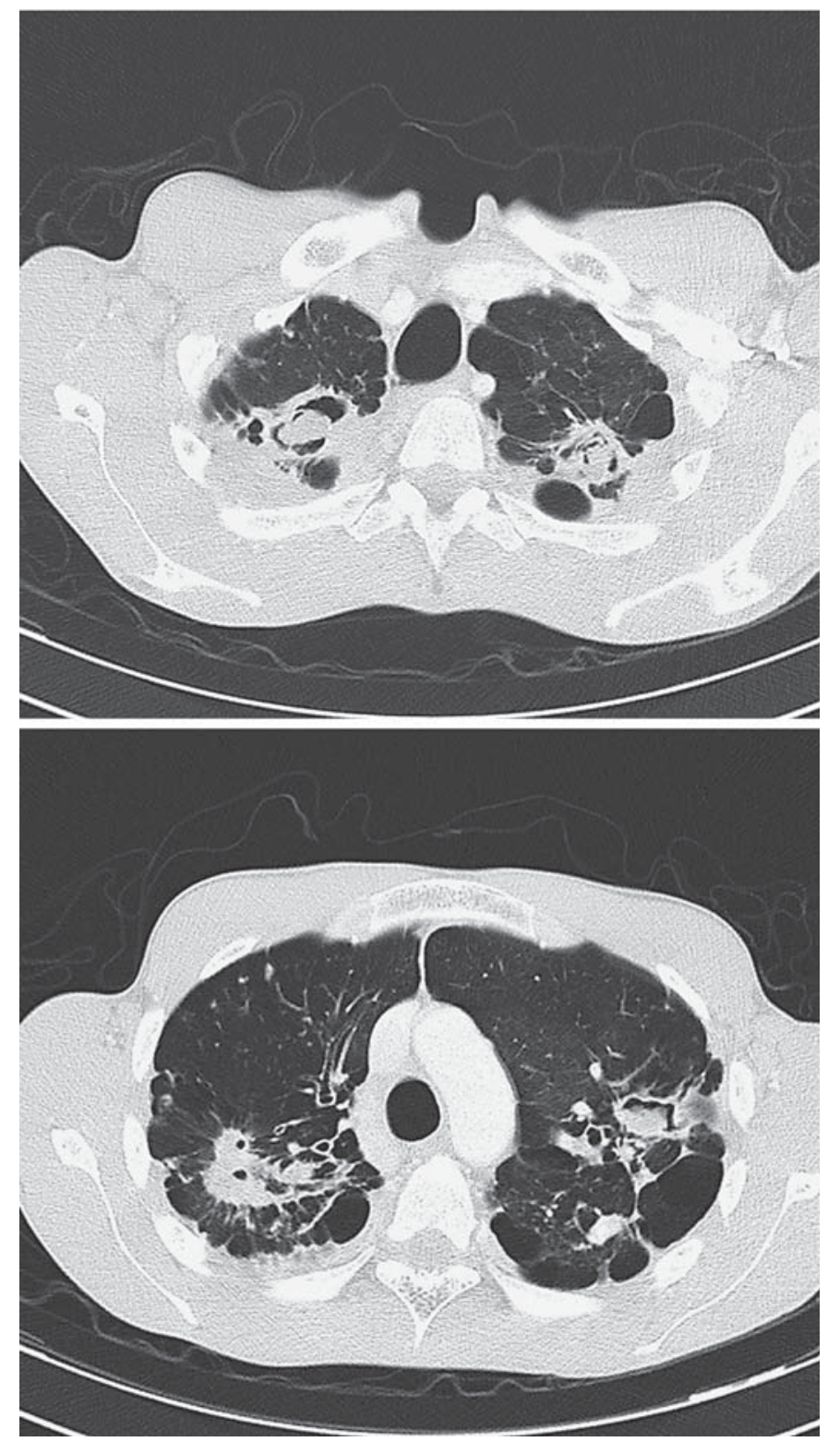

Fig. 2. CT of the chest confirmed marked structural lung disease with UL predominance. Numerous cavities and intracavitary bodies indicating aspergillomata were present.

Our patient had an unremarkable stay in hospital and was discharged without complications. At a follow-up 3 months after the procedure, he reported absolutely no haemoptysis. Moreover, he was subsequently employed as a manual labourer. His pulmonary function testing confirmed significant improvement $\left(\mathrm{FEV}_{1}=\right.$ 1.40 liters $(34.2 \%), \mathrm{FVC}=2.0$ liters $(41.5 \%)$ and 6 -min walking distance $=535 \mathrm{~m})$. This functional improvement was still present at the 6-month follow-up, when he once again reported no stentrelated complications or haemoptysis.

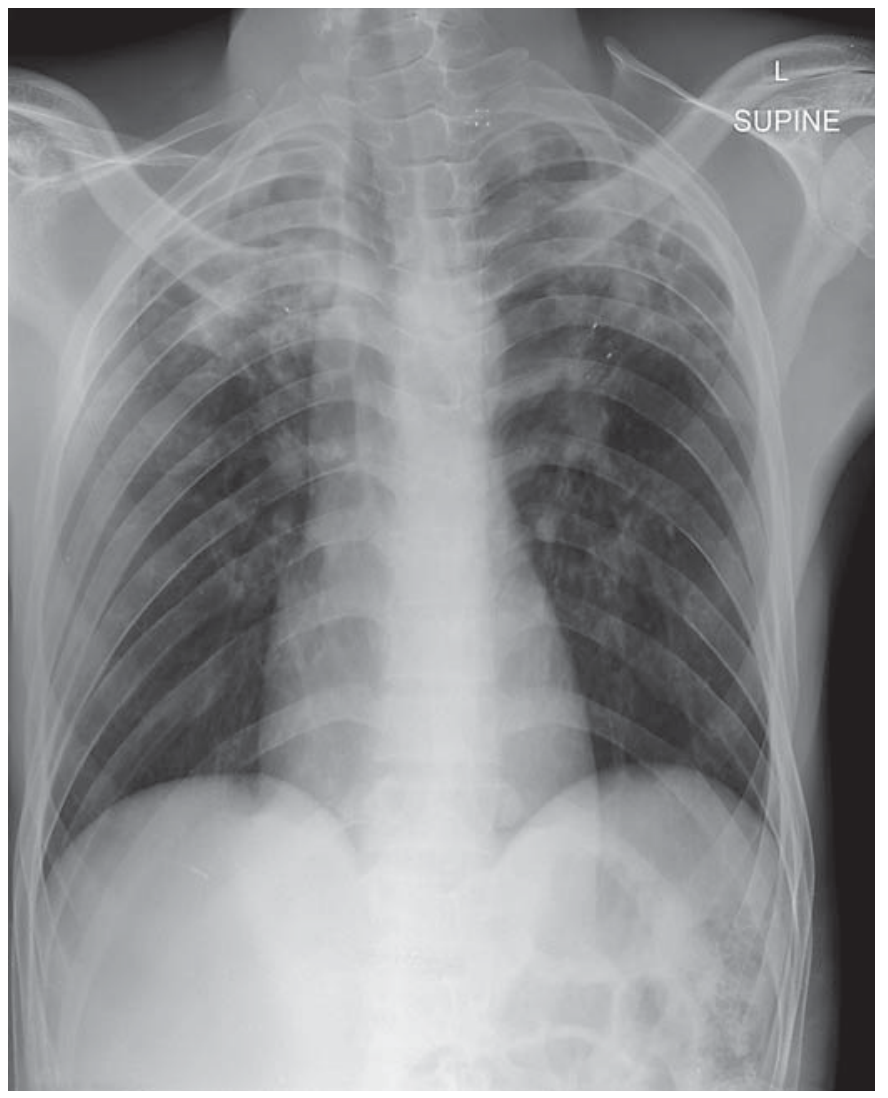

Fig. 3. The patient's chest radiograph immediately after the procedure confirmed adequate stent deployment with partial volume loss of both ULs.

\section{Discussion}

The placement of endobronchial valves in our patient represents, to the best of our knowledge, both a novel indication for the use of these devices and a novel intervention for refractory haemoptysis. Our patent experienced no haemoptysis for a sustained period of 6 months, and significant functional improvements were observed.

Most current available endoscopic interventions for life-threatening haemoptysis necessitate rigid bronchoscopy $[5,6]$. Laser photocoagulation, argon plasma coagulation, electrocautery and the endoscopic instillation of a fibrinogen-thrombin combination, various balloon tamponade devices and even self-expanding airway stents have all been utilised with variable success [6, 13-17]. Endobronchial airway blockade may achieve acute control [6]. Dutau et al. [7] described the endobronchial placement of a silicone spigot, which prevented alveolar inundation and proved adequate for temporary control of 
bleeding, allowing stabilisation prior to endovascular embolisation. Topical haemostatic tamponade therapy, using an oxidized regenerated cellulose mesh, has been used successfully to achieve airway blockade in this setting, as has biocompatible glue [18-20]. In one study, immediate arrest of the haemorrhage was achieved in all but 1 of 57 patients treated with a haemostatic cellulose mesh, all of whom remained free of haemoptysis for 2 days [18]. Six patients subsequently underwent bronchial artery embolisation for recurrence of haemoptysis, and haemostatic tamponade therapy had to be repeated in 2 of the 6 patients. No death secondary to haemoptysis was reported; 5 patients developed post-obstructive pneumonia during a mean follow-up of 10 months [18]. An oxidized regenerated cellulose mesh is absorbed with time, and late recurrence of haemoptysis is therefore a theoretical risk [19]. Endobronchial application of n-butyl cyanoacrylate has only been studied in mild haemoptysis [20]. In one series of 6 cases, short-term control was achieved in 5 patients [20].

Unidirectional endobronchial valves, in essence, function as endobronchial airway blockade devices [21]. Current commercially available devices are designed to allow the drainage of sections and air during expiration, while blocking the entrance of air during inspiration, resulting in complete or partial atelectasis [21]. These devices have been shown to improve quality of life, lung function and exercise capacity in patients with emphysema, particularly when lobar occlusion is achieved, heterogeneous disease is present and collateral ventilation negligible [21-23].

Complications related to valve treatment are relatively rare $(10.3 \%)$ and include pneumonia, haemoptysis and pneumothorax $[22,23]$. We postulated that segmental collapse and haemostasis would result in a functional tamponade, very similar to that achieved with a silicone spigot. Whether or not the intactness of interlobar fissures, collateral circulation and lobar distribution of the underlying cause of haemoptysis are relevant to the successful use of these devices in haemoptysis remain to be elucidated. Only prospective studies would be able to better delineate the potential candidates for endobronchial valves in this setting as well as the success rate, mean time to and rate of haemoptysis recurrence. The possibility of haemorrhage through the endobronchial valve remains a theoretical concern. Potential complications, including pneumonia and haemoptysis, may also be seen as limitations of these devices [21]. Endobronchial valves, as is the case with other blockade devices, will also not be suitable for tracheal and other proximal sites of bleeding and cas- es where lobar or segmental occlusion cannot be tolerated $[6,19]$. Albeit unlikely, the possibility that the patient's ongoing haemoptysis settled spontaneously remains a theoretical possibility.

The extent of the functional improvement observed in our patient was unexpected and remains only partially explained by the partial deflation of his diseased upper lung zones. His $\mathrm{FEV}_{1}$ improved by almost $80 \%$ and his 6-min walking distance by $457 \mathrm{~m}$. The functional improvement observed in the VENT study (both the European and US cohorts) was significantly less [22, 23]. Alternative explanations for the marked improvement may include functional impairment due to an undetected infective exacerbation at the time of the initial evaluation, reduced dynamic hyperinflation with exercise and the cessation of low-grade alveolar inundation of the mid and lower lung zones. The patient's residual volume was not measured prior to the implantation (as improvement in functional parameters was never the primary goal of the procedure), and the exact mechanisms for these improvements are therefore unclear. Furthermore, whether or not comparable improvement in functional capacity and lung function could be observed in other patients with bronchiectasis with a clear lobar predominance remains to be answered.

In conclusion, our case report suggests that endobronchial valves may be a potential adjunct to the interventional pulmonologist's armamentarium used to achieve airway blockade in patients with life-threatening haemoptysis, and that endobronchial valves may improve pulmonary function in patients with bronchiectasis secondary to tuberculosis.

\section{Acknowledgments}

The authors are grateful to Olympus KeyMed (UK), who donated the endobronchial valves.

\section{Financial Disclosure and Conflicts of Interest}

None. 


\section{References}

1 van den Heuvel MM, Els Z, Koegelenberg CF, Naidu KM, Bolliger CT, Diacon AH: Risk factors for recurrence of haemoptysis following bronchial artery embolisation for life-threatening haemoptysis. Int J Tuberc Lung Dis 2007;11:909-914.

-2 Osaki S, Nakanishi Y, Wataya H, Takayama K, Inoue K, Takaki Y, Murayama S, Hara N: Prognosis of bronchial artery embolization in the management of hemoptysis. Respiration 2000;67:412-416

-3 Gross AM, Diacon AH, van den Heuvel MM, Janse van Rensburg J, Harris D, Bolliger CT: Management of life-threatening haemoptysis in an area of high tuberculosis incidence. Int J Tuberc Lung Dis 2009;13:875-880.

$\checkmark 4$ von Groote-Bidlingmaier F, Koegelenberg CF, Bolliger CT: Functional evaluation before lung resection. Clin Chest Med 2011;32:773782.

5 Freitag L, Tekolf E, Stamatis G, Montag M, Greschuchna D: Three years' experience with a new balloon catheter for the management of haemoptysis. Eur Respir J 1994;7:2033-2037.

6 Sakr L, Dutau H: Massive hemoptysis: an update on the role of bronchoscopy in diagnosis and management. Respiration 2010;80:3858.

7 Dutau H, Palot A, Haas A, Decamps I, Durieux O: Endobronchial embolization with a silicone spigot as a temporary treatment for massive haemoptysis: a new bronchoscopic approach of the disease. Respiration 2006;73: 830-832.

$>8$ Springmeyer SC, Bolliger CT, Waddell TK, Gonzalez X, Wood DE: Treatment of heterogeneous emphysema using the spiration IBV valves. Thorac Surg Clin 2009;19:247-253. $\checkmark 9$ Wood DE, McKenna RJ Jr, Yusen RD, Sterman DH, Ost DE, Springmeyer SC, Gonzalez HX, Mulligan MS, Gildea T, Houck WV, Machuzak M, Mehta AC: A multicenter trial of an intrabronchial valve for treatment of severe emphysema. J Thorac Cardiovasc Surg 2007;133:65-73.

10 Hopkinson NS, Toma TP, Hansell DM, Goldstraw P, Moxham J, Geddes DM, Polkey MI: Effect of bronchoscopic lung volume reduction on dynamic hyperinflation and exercise in emphysema. Am J Respir Crit Care Med 2005;171:453-460.

11 Feller-Kopman D, Bechara R, Garland R, Ernst A, Ashiku S: Use of a removable endobronchial valve for the treatment of bronchopleural fistula. Chest 2006;130:273-275.

$>12$ Dooms CA, De Leyn PR, Yserbyt J, Decaluwe $\mathrm{H}$, Ninane V: Endobronchial valves for persistent postoperative pulmonary air leak: accurate monitoring and functional implications. Respiration 2012;84:329-233.

13 Edmondstone WM, Nanson EM, Woodcock AA, Millard FJ, Hetzel MR: Life-threatening haemoptysis controlled by laser photocoagulation. Thorax 1983;38:788-789.

14 Keller CA, Hinerman R, Singh A, Alvarez F: The use of endoscopic argon plasma coagulation in airway complications after solid organ transplantation. Chest 2001;119:1968-1975.

15 Homasson JP: Endobronchial electrocautery. Semin Respir Crit Care 1997;18:535-543.

$>16$ Wong LT, Lillquist YP, Culham G, DeJong BP, Davidson AG: Treatment of recurrent hemoptysis in a child with cystic fibrosis by repeated bronchial artery embolizations and long-term tranexamic acid. Pediatr Pulmonol 1996;22:275-279.
17 Brandes JC, Schmidt E, Yung R: Occlusive endobronchial stent placement as a novel management approach to massive hemoptysis from lung cancer. J Thorac Oncol 2008;3: 1071-1072.

18 Valipour A, Kreuzer A, Koller H, Koessler W, Burghuber OC: Bronchoscopy-guided topical hemostatic tamponade therapy for the management of life-threatening hemoptysis. Chest 2005;127:2113-2118.

19 Reisz G: Topical hemostatic tamponade: another tool in the treatment of massive hemoptysis. Chest 2005;127:1888-1889.

20 Bhattacharyya P, Dutta A, Samanta AN, Chowdhury SR: New procedure: bronchoscopic endobronchial sealing; a new mode of managing hemoptysis. Chest 2002;121:20662069.

21 Gasparini S, Zuccatosta L, Bonifazi M, Bolliger CT: Bronchoscopic treatment of emphysema: state of the art. Respiration 2012;84: 250-263.

22 Sciurba FC, Ernst A, Herth FJF, Strange C, Criner GJ, Marquette CH, Kovitz KL, Chiacchierini RP, Goldin J, McLennan G: A randomized study of endobronchial valves for advanced emphysema. N Engl J Med 2010; 363:1233-1244.

-23 Herth FJF, Noppen M, Valipour A, Leroy S, Vergnon JM, Ficker JH, Egan JJ, Gasparini S, Agusti C, Holmes-Higgin D, Ernst A: Efficacy predictors of lung volume reduction with Zephyr valves in a European cohort. Eur Respir J 2012;39:1334-1342.

24 Gompelmann D, Eberhardt R, Herth FJ: Collateral ventilation. Respiration 2013;85:515520 . 